\title{
Entanglement detachment in fermionic systems
}

\author{
Hernán Santos, ${ }^{1,2}$ José E. Alvarellos, ${ }^{1}$ and Javier Rodríguez-Laguna ${ }^{1}$ \\ ${ }^{1}$ Dep. de Física Fundamental, Universidad Nacional de Educación a Distancia (UNED), Madrid, Spain \\ ${ }^{2}$ Dep. de Física de la Materia Condensada, Universidad Autónoma de Madrid, Cantoblanco, 28049 Madrid, Spain
}

(Dated: September 18, 2018)

\begin{abstract}
This article introduces and discusses the concept of entanglement detachment. Under some circumstances, enlarging a few couplings of a Hamiltonian can effectively detach a (possibly disjoint) block within the ground state. This detachment is characterized by a sharp decrease in the entanglement entropy between block and environment, and leads to an increase of the internal correlations between the (possibly distant) sites of the block. We provide some examples of this detachment in free fermionic systems. The first example is an edge-dimerized chain, where the second and penultimate hoppings are increased. In that case, the two extreme sites constitute a block which disentangles from the rest of the chain. Further examples are given by (a) a superlattice which can be detached from a 1D chain, and (b) a star-graph, where the extreme sites can be detached or not depending on the presence of an external magnetic field, in analogy with the Aharonov-Bohm effect. We characterize these detached blocks by their reduced matrices, specially through their entanglement spectrum and entanglement Hamiltonian.
\end{abstract}

\section{INTRODUCTION}

Quantum many-body physics constitute a theoretical crossroad between condensed matter physics and quantum information, since both of them benefit from the study of correlations and entanglement structures [1, 2]. Indeed, the ground states (GS) of quantum systems present very interesting entanglement properties. Under a variety of circumstances, they follow the area law [3], which determines that the entanglement entropy of a region is proportional to the measure of its boundary. The area law holds for 1D gapped systems [4], but some 1D gapless systems are known to violate it, such as the rainbow system [5] 7, a free fermionic chain with exponentially decaying hoppings as we move away from the center, which presents maximal entanglement between the left and right halves in its GS.

In this work we will characterize systems where the increase of some couplings leads to a block effectively detaching from the system within its GS. Thus, as those couplings grow we observe a sharp decline in the entanglement entropy between the block and its environment. The block in question need not be formed by contiguous sites. Effectively, in a first example, the block can be constituted by the two extreme sites of an open freefermionic chain, and the selected couplings will be the second and penultimate hoppings 8. A further consequence is that, through entanglement monogamy [9], the sites of the block become more correlated among themselves, even if they are far away. In our two other examples the detached block is given by a superlattice of a chain and the edge sites of a star-graph, always for a free-fermion system. Also in these cases, the block sites establish large correlations, despite being far away.

When the entanglement entropy between block and environment falls to zero the GS can be effectively factorized into two wavefunctions, one for the block and the other for the environment. For low entanglement entropy, but still non-zero, the best way to characterize physics on the block is still to analyze its reduced density matrix. Indeed, we have considered in all the relevant cases the entanglement spectrum [10] and the entanglement Hamiltonian [11 13. In the studied cases, we show that both the superlattice and the edge sites of the stargraph constitute an effective 1D free-fermionic system. The case of the star-graph presents special relevance because whether the edge sites detach or not depends on the number of legs of the star, modulo 4. Moreover, an external magnetic field can re-connect the edge sites to the rest of the system, or detach them again, in a way which is reminiscent of the Aharonov-Bohm effect.

This work is a continuation of our previous article [8, where we proposed an strategy to optimize the correlation between the extreme sites of a chain, while keeping the energy gap constant. The optimal strategy found was, precisely, to increase certain hoppings, presenting an intriguing similarity with the Su-Schrieffer-Heeger (SSH) model of a dimerized fermionic chain 14 16, which is known to present a non-trivial symmetry protected topological phase 17. In this case, the large correlations pattern is extended to several sites, in more complex configurations.

This article is organized as follows. Sec. II presents the model for independent fermions. In Sec. III we summarize the behavior of the edge-dimerized chains, taken from 8. Superlattices built from edge-dimerized chains are the focus of Sec. IV] Afterwards, in Sec. V] we discuss junctions with a central ring, which present in some cases a topological obstruction to the detachment, which can be removed using a magnetic flux. Our conclusions are summarized in the last Section.

\section{MODEL}

Let us consider a chain of $L$ sites and $N_{e}$ independent spinless Dirac fermions. A tight-binding Hamiltonian can 
be written in the following way:

$$
H=-\sum_{i} t_{i} c_{i}^{\dagger} c_{i+1}+\text { h.c. }
$$

where $c_{i}^{\dagger}$ is the creation operator at site $i$ and the $t_{i}$ are the local hopping amplitudes. Unless otherwise stated, we will consider $N_{e}=L / 2$, i.e., half-filling.

The ground state (GS) of (1) can always be written as a Slater determinant:

$$
|\Psi\rangle=\prod_{k=1}^{N_{e}} b_{k}^{\dagger}|0\rangle
$$

with $|0\rangle$ the Fock vacuum and $b_{k}^{\dagger}$ the creation operators for the orbitals, given by a canonical transformation

$$
b_{k}^{\dagger}=\sum_{i} U_{k i} c_{i}^{\dagger}
$$

where $U$ is the matrix that diagonalizes the hopping matrix, $T_{i j}=t_{i}\left(\delta_{i, i+1}+\delta_{i, i-1}\right)$, with eigenvalues $\varepsilon_{k}$. The energy gap of the system is given by the minimal excitation energy, $\varepsilon_{(L / 2)+1}-\varepsilon_{L / 2}$.

The correlation matrix, defined as

$$
C_{i, j}=\left\langle\Psi\left|c_{i}^{\dagger} c_{j}\right| \Psi\right\rangle .
$$

allows us to compute the expectation value of any observable on any state given by Eq. 22, via Wick's theorem. It can be evaluated using the matrix $U$ :

$$
C_{i, j}=\sum_{k=1}^{N_{e}} \bar{U}_{k i} U_{k j}
$$

Entanglement properties of a generic block of the chain, $B=\left\{i_{1}, \cdots, i_{\ell}\right\}$ (note that the sites $i_{1}, \cdots, i_{\ell}$ are possibly disjointed), are always referred to the reduced density matrix of $|\Psi\rangle$, defined as

$$
\rho^{B} \equiv \operatorname{Tr}_{B}|\Psi\rangle\langle\Psi|,
$$

being $\operatorname{Tr}_{B}$ the partial trace on the block. In the case of a Slater determinant, this $\rho^{B}$ can be expressed as a tensor product of $2 \times 2$ density matrices of the form [11]

$$
\rho^{B}=\bigotimes_{k=1}^{\ell}\left(\begin{array}{cc}
\nu_{k}^{B} & 0 \\
0 & 1-\nu_{k}^{B}
\end{array}\right) .
$$

where the $\nu_{k}^{B} \in[0,1]$ are the eigenvalues of the correlation $\ell \times \ell$ sub-matrix corresponding to the block $C^{B}$ (i.e., those elements of $C_{i, j}$ with $i, j \in B$ ).
These eigenvalues can be interpreted as occupation numbers for fermionic modes, associated to a set of entanglement energies $\epsilon_{k}^{B}$ given by

$$
\nu_{k}^{B}=\frac{1}{1+\exp \epsilon_{k}^{B}} .
$$

These values $\left\{\epsilon_{k}^{B}\right\}$ constitute the so-called entanglement spectrum (ES) [10, which make up the single-body energies of the entanglement Hamiltonian $(\mathrm{EH}), H^{B}$, defined as the Hamiltonian whose thermal state at $\beta=1$ corresponds to the actual reduced density matrix,

$$
\rho^{\mathcal{B}}=\exp \left(-H^{B}\right) .
$$

For free fermionic states, the EH is a quadratic Hamiltonian with a new effective hopping matrix which can be obtained from the correlation matrix 12 . The entanglement gap is defined as the energy gap of $H^{B}$, $\epsilon_{(L / 2)+1}^{B}-\epsilon_{L / 2}^{B}$.

On the other hand, the entanglement entropy of the block, defined as the von Neumann entropy of $\rho^{B}$,

$$
S^{B}=-\operatorname{Tr} \rho^{B} \log \rho^{B},
$$

can be computed using the following expression [1]

$$
S^{B}=-\sum_{k=1}^{\ell}\left(\nu_{k}^{B} \log \nu_{k}^{B}+\left(1-\nu_{k}^{B}\right) \log \left(1-\nu_{k}^{B}\right)\right) .
$$

Our interest in the aforementioned entanglement measures stems from the fact that they are usually able to characterize the different phases of matter through e.g. corrections of (or violations to) the area law for the entanglement entropy [6, 18.

Beyond the entropy, the ES and EH provide a complete characterization of the dynamics of a block as a mixed state embedded in the ground state of the total system. The EH can be regarded as the effective Hamiltonian describing the state of the block. Thus, the EH of a disjoint block can provide an estimate of the effective couplings between different regions of the system.

\section{EDGE-DIMERIZED CHAIN}

Our first example of entanglement detachment is provided by the edge-dimerized chain, described in [8] as a nearly optimal way to obtain large end-to-end correlations with a robust energy gap. Consider an open fermionic chain of $L$ (even) sites, subject to the Hamiltonian (1), with the hoppings $t_{i}$ given by

$$
t_{i}= \begin{cases}1 & \text { if } i \neq 2, L-1 \\ t_{0} & \text { if } i=2 \text { or } i=L-1\end{cases}
$$




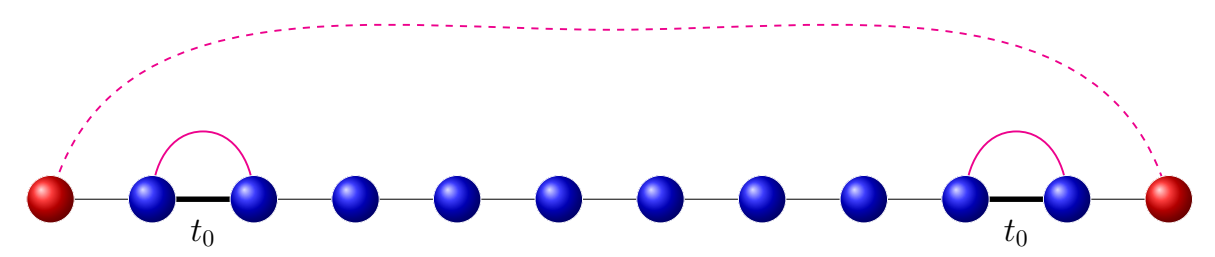

FIG. 1. Illustration for the edge-dimerized chain. The second and penultimate hoppings are set to a different value than the rest, $t_{0}$. If $t_{0} \gg 1$, a valence bond is set on top of these links, thus forcing a long-distance valence bond between the first and last sites.

i.e.: only hoppings $t_{2}=t_{L-1}=t_{0}$ are distinguished, as illustrated in Fig. 1. If $t_{0} \gg 1$, a valence bond will appear on top of links 2 and $L-1$, i.e. between sites 2 and 3 and between sites $L-2$ and $L-1$, thus leaving sites 1 and $L$ no option but to establish a valence bond themselves. Otherwise, if $t_{0} \ll 1$, the chain effectively decouples: the block containing sites $\{1,2\}$ and the block containing sites $\{L-1, L\}$ become isolated from the rest of the chain.

Fig. 2 shows the correlation between sites 1 and $L$, or end-to-end correlation, $\left|C_{1, L}\right|$, as a function of $t_{0}$ for different values of $L$, within the GS of Hamiltonian (1). We can see that, for low $t_{0}$, the correlation grows as $t_{0}^{2}$, as discussed theoretically in [8, while it saturates to $1 / 2$ for $t_{0} \rightarrow \infty$. This large value of the correlation implies that a valence bond has been created between these two extreme sites. In other words, they have established a Bell pair, which is maximally entangled. Thus, through the monogamy of entanglement, the block constituted by sites $B=\{1, L\}$ should detach from the rest of the chain. In Fig. 2 we can also see the entropy of this block, $S^{B}$, as a function of $t_{0}$. Indeed, we observe that for very low $t_{0}, S^{B} \approx 2 \log 2$, which is the maximal possible entanglement. As we increase $t_{0}$, the entropy decreases steadily, tending to zero for large $t_{0}$. Thus, sites 1 and $L$ become effectively detached, and the global wavefunction becomes factorizable.

In Fig. 2 we have also plotted the energy gap, $\Delta E$ as a function of $t_{0}$ for different values of $L$. The energy gap is a measure of the robustness of the ground state. We can observe that the energy gap falls as $\Delta E \sim t_{0}^{-2}$, as argued in [8].

This example serves as an illustration of the general phenomenon, which will be discussed in more interesting situations in the following sections: as a few couplings are increased, a certain block may become detached from the rest of the system, i.e.: the entanglement between the block and the rest of the system will fall to zero.

\section{SUPERLATTICE CHAIN}

Let us introduce a more interesting situation, where entanglement detachment takes place for a disconnected block making up a superlattice whose sites are periodi-

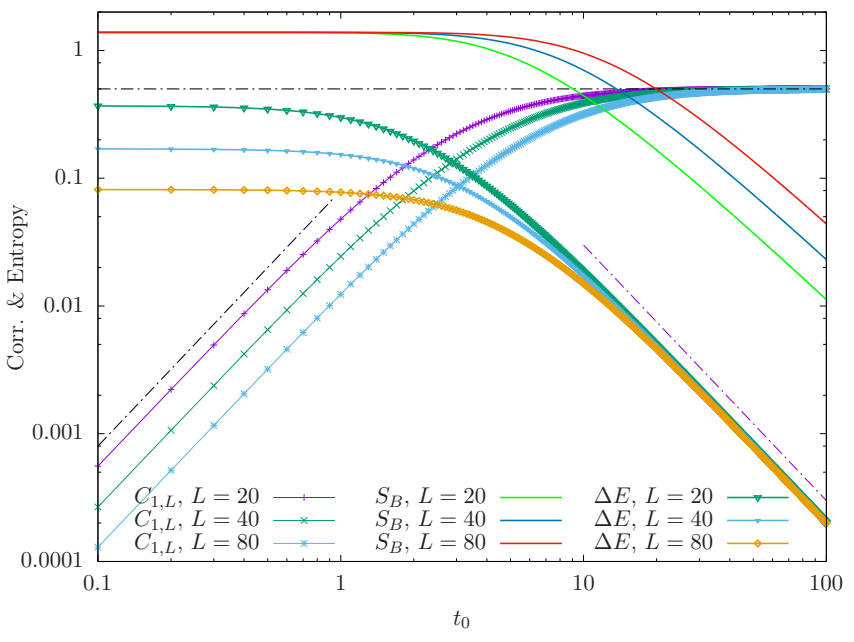

FIG. 2. End-to-end correlation, $\left|C_{1, L}\right|$, energy gap, $\Delta E$, and entanglement entropy $S^{B}$ of the block $B=\{1, L\}$ as a function of $t_{0}$ in the Hamiltonian (1) with hoppings given by Eq. (12). The marked dashed lines correspond to scalings $t_{0}^{2}$ and $t_{0}^{-2}$. The horizontal line marks the limit correlation of $1 / 2$.

cally spaced along the chain. As we will show, the superlattice can be effectively disentangled from the rest of the system by enhancing a few selected hoppings.

Let us consider a periodic chain of $L$ sites, with $L=$ $\ell \times m$, where $m$ will be the number of sites in the superlattice block and $\ell$ will be the distance between them, as illustrated in Fig. 3 using $m=3$ and $\ell=7$. We will focus on the case when both $m$ and $\ell$ are an odd numbers. Thus, $L$ is an odd number and it is impossible to achieve a half-filling occupation. We will then consider the system with $N_{e}=(L-1) / 2$ fermions. The superlattice sites will be denoted by $B=\left\{s_{1}, \cdots, s_{m}\right\}$, where $s_{j}=(j-1) m$.

The system will also be subject to Hamiltonian (1), with all hoppings set to $t_{i}=1$ except a few selected ones enhanced to $t_{i}=t_{0}$. These selected hoppings are the second and penultimate of each segment between superlattice sites, and they are denoted by thick black lines in Fig. 3. When $t_{0} \gg 1$, these hoppings are strong and a valence bond state tends to be placed upon them, thus forcing the superlattice sites to live in relative isolation and inducing them to establish large correlations among 


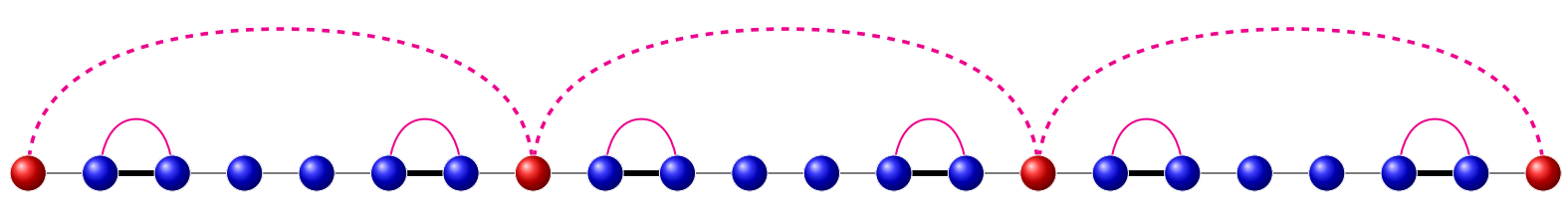

FIG. 3. Illustration of the system used to detach a superlattice from the rest of the chain, inducing large multiparty correlations among the superlattice sites. The chain has periodic boundaries (the last site is the same as the first) and presents regular (thin black lines) and strong (thick black lines) links. The red sites, which make up the superlattice, are surrounded by sites which form strong bonds, thus effectively separating them from the rest of the chain. The strong links will tend to form a valence bond (red lines). Large distance correlations between the superlattice sites may appear (as indicated with dashed red lines). In this example, we have $m=3$ superlattice sites (remember that first and last sites are the same) separated by $\ell=7$ hoppings. The number of sites is $L=m \times \ell=21$ in this case.

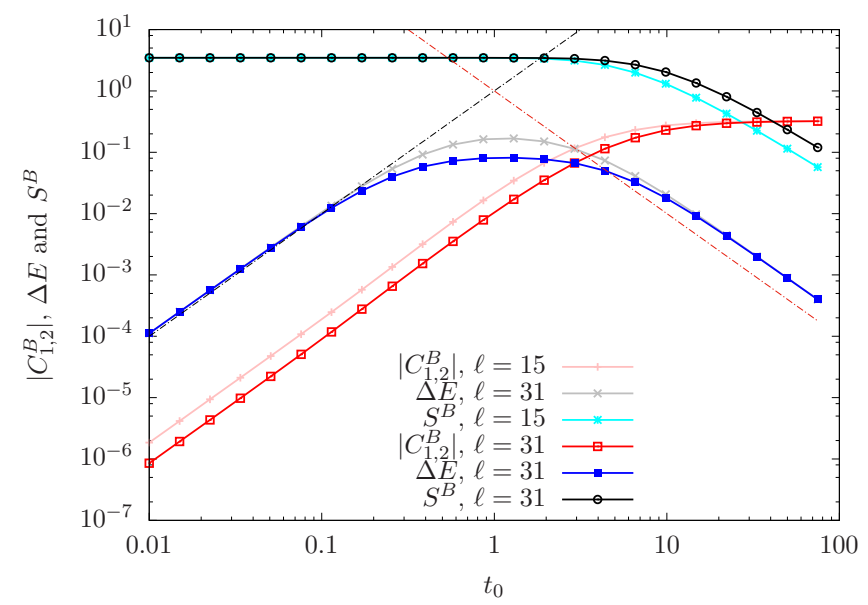

FIG. 4. Superlattice system with $m=5$ and $\ell=15$ or 31 , i.e. with $L=75$ or 155 sites. The enhanced hopping term is given by $t_{0}$. The correlation between neighboring superlattice sites (those in red in Fig. 3 $\left|C_{1,2}^{B}\right|$ increases like $t_{0}^{2}$ for low $t_{0}$, and saturate at a value $\sim 0.32$ for both system sizes. The energy gap, $\Delta E$, can be seen to grow as $t_{0}^{2}$ for low $t_{0}$ and decrease like $t_{0}^{-2}$ for large $t_{0}$, having a maximum at $t_{0}=1$. The block entropy of the superlattice sites, $S^{B}$ decreases from its maximum possible value for $t_{0} \rightarrow 0^{+}, 5 \log (2) \sim 3.46$, as $t_{0}^{-2}$ when $t_{0}>1$.

themselves. Let us consider the submatrix $C^{B}$, denoted by

$$
C_{i, j}^{B}=\left\langle c_{s_{i}}^{\dagger} c_{s_{j}}\right\rangle,
$$

on the ground state. Notice that, due to the periodic boundary conditions, $C_{i, j}^{B}$ only depends on $|i-j|$. Using Eq. (11) we can obtain the entanglement entropy between the superblock and the rest of the system. As we will show, the superlattice effectively detaches from the rest of the system for large values of $t_{0}$.

We present in Fig. 4 the correlation between neighboring superlattice sites, $\left|C_{1,2}^{B}\right|$, as a function of $t_{0}$ for a system with $m=5$ and different values of $\ell$ (15 and 31). We notice that if $t_{0}<1$ the correlation grows steadily as $t_{0}^{2}$, and it saturates for both values of $\ell$ at the same value, close to $\left|C_{1,2}^{B}\right| \sim 0.32$. Fig. 4 also provides information about the energy gap, $\Delta E$, which can be seen to decay both for large and small values of $t_{0}$, like $t_{0}^{-2}$ and $t_{0}^{2}$ respectively, with a maximum at $t_{0}=1$.

The last magnitude shown in Fig. 4 is the superlattice block entropy, $S^{B}$, associated to the partition between the superlattice and the rest of the system. We can see that when $t_{0} \lesssim 1$ the entropy $S^{B}$ takes its maximum possible value, $5 \log (2) \approx 3.46$ (because $n \log (2)$ corresponds to $n$ bond cuts, see [6]), showing that for such low values of $t_{0}$ there is no connection between the superlattice sites. But for $t_{0}>1$ the block entropy starts to decrease, also with a $t_{0}^{-2}$ behavior, marking the beginning of the slow detachment of the superlattice block from the rest of the system.

Remarkably, the correlation, the gap, and the entropy of the block of superlattice sites, are rather independent of the system size. Moreover, we notice that the entanglement detachment, as measured by the superlattice entropy, only takes place when the correlation $\left|C_{1,2}^{B}\right|$ is nearly saturated.

\section{A. Entanglement Hamiltonian}

As the superlattice gets more and more detached, it becomes relevant to ask what is the effective Hamiltonian describing the subsystem. As we know, the $\mathrm{EH}$ is an effective way to describe a certain subsystem when we do not have access to the rest of the system, see Eq. (9). For a system of independent fermions, the $\mathrm{EH}$ is a quadratic Hamiltonian in the fermionic fields [11-13 and, due to the periodic structure of our system, its elements must be translation invariant. Thus, we can write

$$
H^{B}=-\sum_{i, j} T(|i-j|) c_{s_{i}}^{\dagger} c_{s_{j}}+\text { h.c. }
$$

where $T(|i-j|)$ denote the effective hopping terms between the superlattice sites (not only nearest neighbors), which would contain the whole information of the superlattice subsystem. The top panel of Fig. 5 shows these matrix elements, $T(|i-j|)$, as a function of $t_{0}$ for the same superlattice system as before. Notice that $T(0)$ plays the role of a chemical potential, while $T(1)$ is the usual 

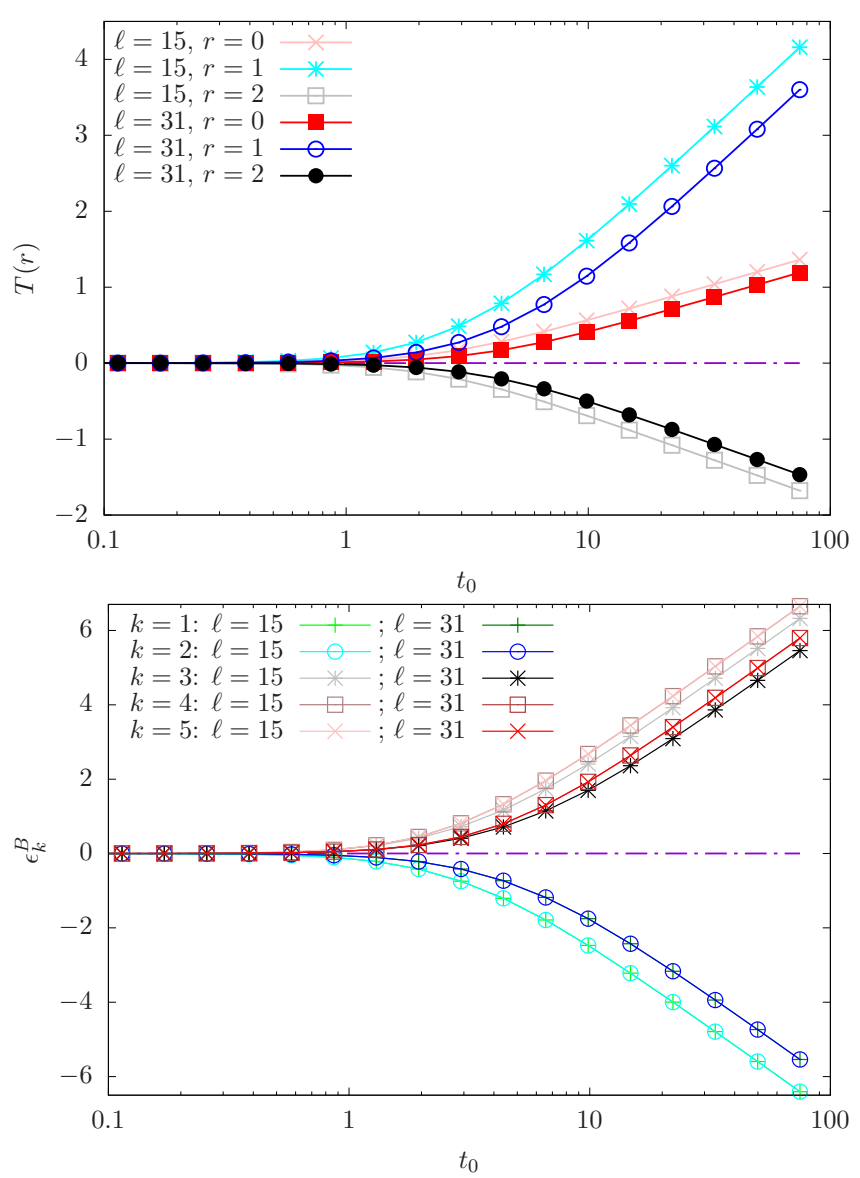

FIG. 5. Top: Entanglement Hamiltonian effective hoppings, $T(r)$ with $r=|i-j|$ (see Eq. 14) for the superlattice block with $m=5$ and $\ell=15$ and 31, as described in Sec. IV] Notice that the effective hoppings grow logarithmically with $t_{0}$ for large values of $t_{0}$. Bottom: entanglement spectrum (ES) of the superlattice block, $\epsilon_{k}^{B}$, as a function of $t_{0}$ for the same two systems. Notice the exact degeneracy of the first two levels, $k=1,2$ and the last two, $k=4,5$.

nearest-neighbor hopping term, while $T(r)$ for $r \geq 2$ become non-local hopping terms, which is non-negligible. All the terms present a logarithmic growth for large $t_{0}$.

The entanglement spectrum (ES) also provides more complete information about the entanglement structure 10. Indeed, it has been widely used in order to detect non-trivial topological phases [10, 19, 20]. The singlebody ES of the superlattice block is represented in the bottom panel of Fig. 5. The $\epsilon_{k}^{B}$, which constitute the single-body spectrum of Hamiltonian (14), grow logarithmically with $t_{0}$ for large $t_{0}$. The two lowest energy levels and the two highest ones are exactly degenerate.

Notice that both the EH and the ES describe the reduced density matrix of the superlattice block as if it corresponded to an effective free-fermionic system at temperature $\beta=1 /\left(k_{B} T\right)=1$. But, alternatively, we may extract an effective temperature as a prefactor for large values of $t_{0}$,

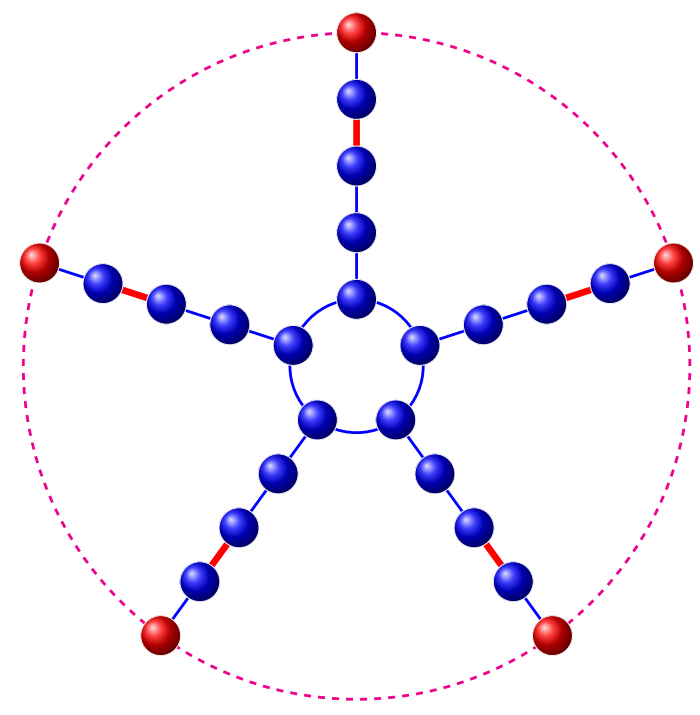

FIG. 6. Illustration of a ring-junction with $n=5$ legs and $\ell=5$ sites per leg. The second link from the edge of all legs (in red) is enhanced, with a value $t_{0}>1$; all other links have a unit coupling. The edge sites (in red), even they are not physically connected, will establish large correlations among themselves (as indicated with red dashed lines).

$$
\rho^{B}\left(t_{0}\right)=\exp \left(-H^{B}\left(t_{0}\right)\right) \sim \exp \left(-\log \left(t_{0}\right) H_{\text {eff }}^{B}\right),
$$

where $H_{\text {eff }}^{B}$ is expected to be independent of $t_{0}$, and corresponds to a limit Hamiltonian. This scaling relation considers the entanglement Hamiltonian as a fixed operator, and the dependence on $t_{0}$ is attached to an effective temperature,

$$
k_{B} T_{\text {eff }}=\frac{1}{\log t_{0}},
$$

Thus, for very large $t_{0}, T_{\text {eff }} \rightarrow 0$ and the superlattice becomes disentangled from the rest of the system.

\section{EDGE-DIMERIZED RING-JUNCTIONS}

Let us consider the ring-junction illustrated in Fig. 6 . The fermionic sites are arranged in $n$ chains of $\ell$ sites joined through one extreme, making up a central ring. All hoppings will take unit value, except the second link starting from the free edge (marked in red), which will take a value $t_{0}$. Now let us consider the block formed by the free extremes of each leg (marked in red), $B=$ $\left\{s_{1}, \cdots, s_{n}\right\}$. We will show that this edge block detaches from the rest of the system when $t_{0}$ becomes very large, while it establishes large internal correlations despite the lack of physical hoppings among them.

Please note that the sites making up the edge block $B$ (marked in red in Fig. 6) are physically disconnected. 

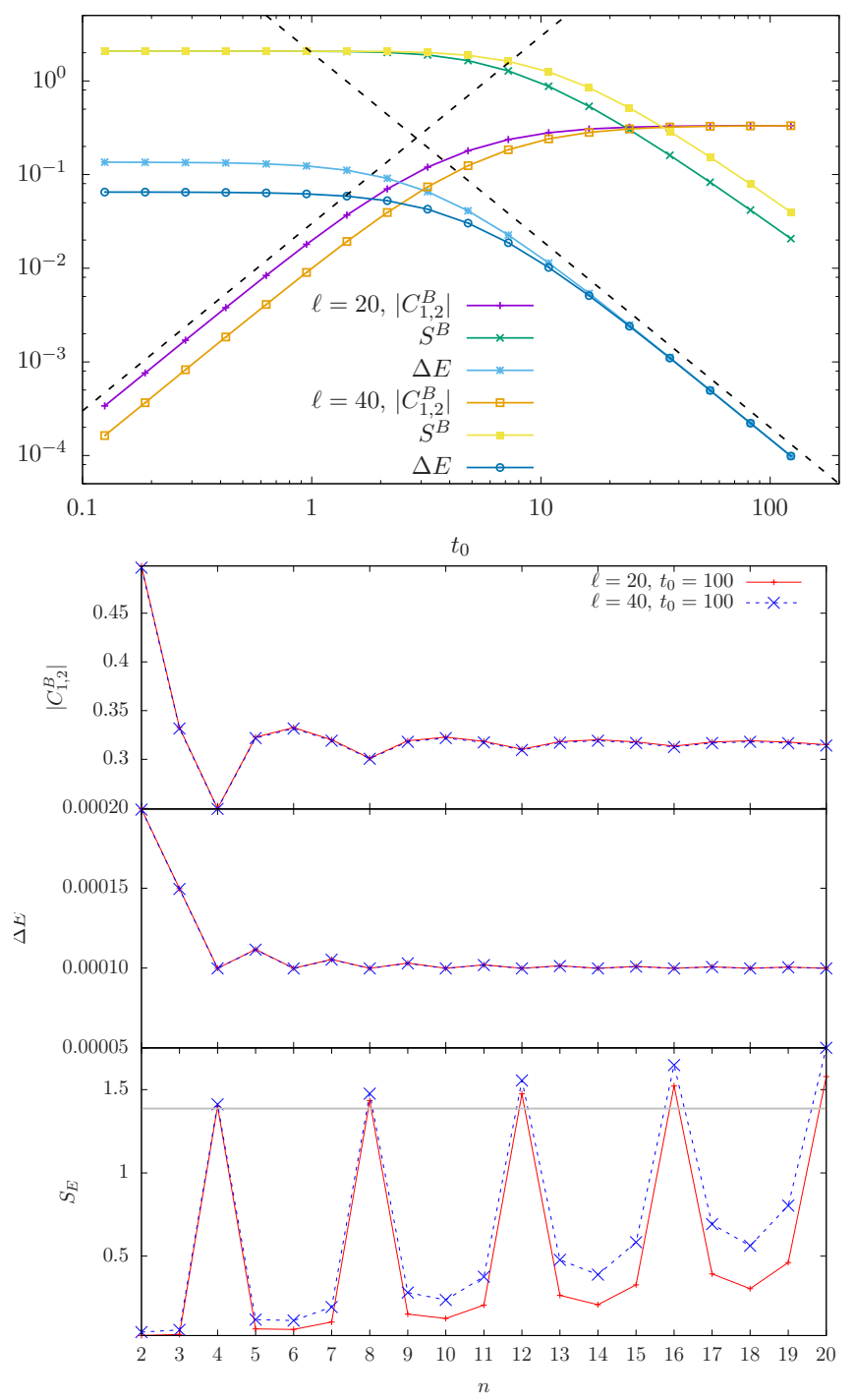

FIG. 7. Top: For an edge-dimerized ring-junction with $n=3$, $\ell=20$ and 40 , we show the correlation between edge sites corresponding to neighboring legs, $\left|C_{1,2}^{B}\right|$, the entropy of the edge block, $S^{B}$, and the energy gap of the GS, all of them as a function of $t_{0}$. Notice the straight lines, which correspond to scalings $t_{0}^{-2}$ and $t_{0}^{2}$. Bottom: same magnitudes for edgedimerized ring-junctions as a function of the number of legs, $n$, for a fixed value of $t_{0}=100$. Notice the periodic pattern in the entropy, $S^{B}$, where the horizontal line marks the value $2 \log (2)$.

For a particle to jump from one to any other, it must hop along one leg in order to reach the center, and then along another leg, in order to reach the new extreme, making a total of $2 \ell-1$ hoppings. Nonetheless, as we shall show, effective hopping terms between them will appear in the entanglement Hamiltonian.

Let $C^{B}$ be the correlation sub-matrix corresponding to the edge block, such that $C_{1,2}^{B}$ stands for the correlation between edge sites of neighboring legs. The top panel of Fig. 7 shows the dependence of this correlation $\left|C_{1,2}^{B}\right|$ on the designated hopping, $t_{0}$, for a ring-junction with $n=3$ legs and two leg sizes: $\ell=20$ and $\ell=40$. We observe, as in the other cases, the $t_{0}^{2}$ dependence for low $t_{0}$, with a saturation at a finite value a bit less than $1 / 2$. The top panel of Fig. 7 also shows the energy gap, $\Delta E$ and the block entropy $S^{B}$ as a function of $t_{0}$ for the same systems. We notice these two magnitudes decrease steadily for large $t_{0}$. Thus, we make our main claim: the edge of the ring-junction detaches for large values of $t_{0}$.

A very relevant effect shows up when we consider different values for the number of legs, $n$, as shown in the bottom panel of Fig. 7 using always $t_{0}=100$, which is close enough to the strong coupling limit, when $t_{0} \rightarrow \infty$. The top plot shows the behavior of the correlation $\left|C_{1,2}^{B}\right|$, where we observe oscillations with $n$ which attenuate for large $n$, around a value $\left|C_{1,2}^{B}\right| \sim 0.314$, i.e., quite high. The energy gap, $\Delta E$, is shown in the second plot, also as a function of $n$. In this case we also observe some oscillations which attenuate for large $n$ around a limit value. The most intriguing situation shows up when we study the entanglement entropy of the edge block, $S^{B}$, as shown in the third plot. There we can see that the oscillation becomes much more marked, and does not attenuate. Besides a certain secular upwards trend, all values of $n$ which are multiples of 4 present a finite entanglement entropy $S^{B}$ which is close to $2 \log (2)$ (marked with the horizontal line). Thus, we observe that, if the number of legs $n=4 k$, the edge block does not detach.

In order to explain this unexpected periodic behavior, we will consider the entanglement spectrum (ES) and entanglement Hamiltonian (EH). We will assume that the $\mathrm{EH}$ presents the same form provided for the superlattice, Eq. (14), since it must be a free-fermion Hamiltonian with translation invariance. In the top panel of Fig. 8 we show $T(r)$ for the first values of $r$ using two systems with $\ell=40$ and two different numbers of legs, $n=12$ and $n=$ 14 , as a function of $t_{0}$. The most salient feature is that $T(0)=T(2)=0$. All even terms are zero. Moreover, we can observe that, for large $t_{0}$, they grow like $\log \left(t_{0}\right)$, as it was the case for the superlattice, see Sec. IV]

The bottom panel of Fig. 8 presents the entanglement energies, $\epsilon_{k}^{B}$ for the same systems. The scaling behavior is very similar, they grow as $\log \left(t_{0}\right)$ for large $t_{0}$. Yet, there is a crucial difference: the case $n=12$ presents an exact zero mode, i.e. $\epsilon_{n / 2}^{B}=\epsilon_{n / 2+1}^{B}=0$ exactly. The same is true for all other $n=4 k$.

Thus, we can still make similar claims to the superlattice case: there is a scaling entanglement Hamiltonian, $H_{\text {eff }}^{B}$, such that, for large enough $\log \left(t_{0}\right)$,

$$
H^{B}\left(t_{0}\right) \sim \log \left(t_{0}\right) H_{\mathrm{eff}}^{B},
$$

But, interestingly, when $n=4 k$, the GS of $H_{\text {eff }}^{B}$ presents exact degeneracy of the ground state. This is a hallmark of a non-trivial topological phase [19, 20, leading to a minimal entanglement entropy, which is given by

$$
S_{\min }=d \log (2),
$$



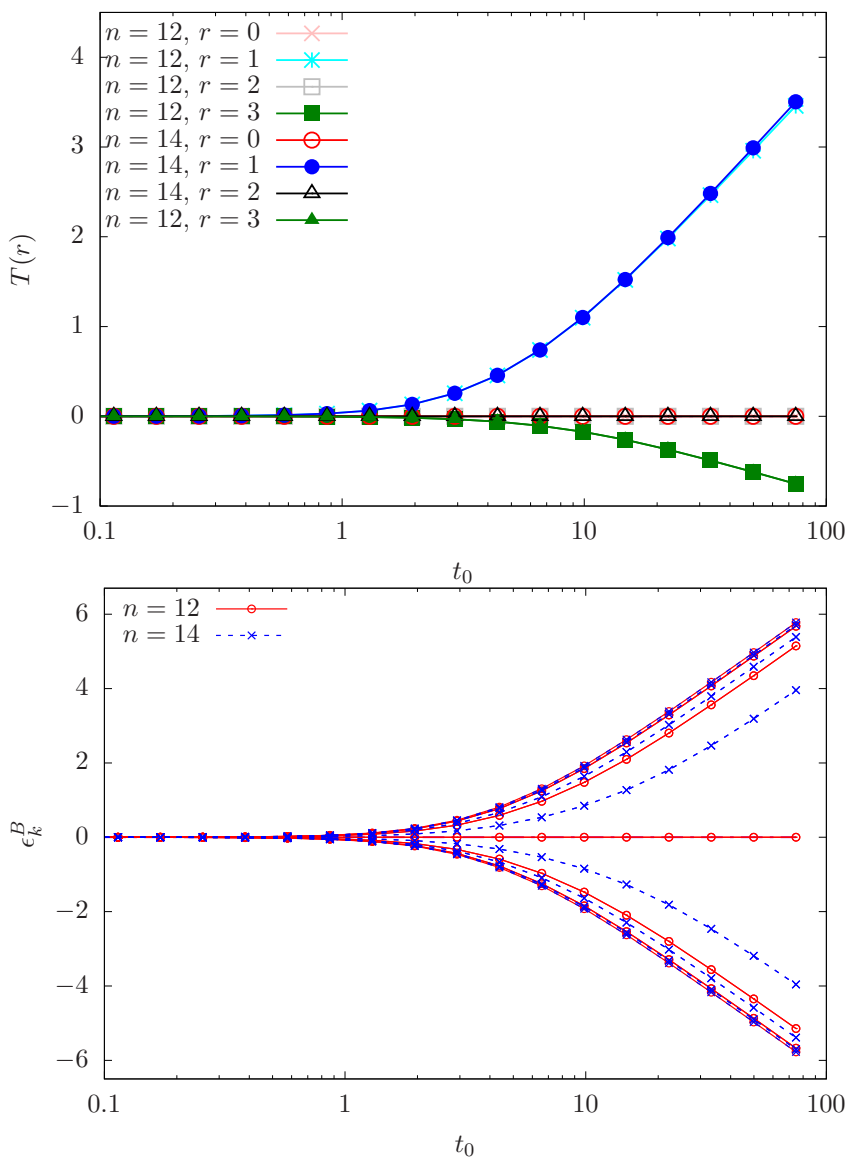

FIG. 8. Top: Entanglement Hamiltonian coefficients, $T(r)$, for edge-dimerized ring-junctions with $\ell=40$ sites per leg, $n=12$ and $n=14$ legs, as a function of $t_{0}$ for the first few values of $r$. Notice that, for large $t_{0}$, they grow like $\log \left(t_{0}\right)$. Moreover, the coefficients present very small differences from $n=12$ to $n=14$. Bottom: Entanglement spectrum of the same systems. Notice the same scaling behavior, with one exception: the ES of the case $n=12$ presents two zero modes, $\epsilon_{n / 2}^{B}=\epsilon_{n / 2+1}^{B}=0$.

where $d$ is given, in our case, by the number of zero modes. Thus, $S_{\min }=2 \log (2)$, in correspondence with the observed behavior of the entanglement entropy for large $t_{0}$ in Fig. 7. In other terms: for $n=4 k$ the edge block does not detach because it presents a minimal entropy of topological origin.

This strong periodicity effect on the number of legs presents an intriguing relation to Hückel's rule for aromaticity, which establishes that for planar rings of carbon atoms, only those with $4 k+2$ atoms can be stable, due to the fact that $\pi$ orbital electrons can be delocalized only if the plane ring has $4 k+2 \pi$ electrons. In our edge-dimerized junctions the edges will be isolated or not from the rest of the system depending on the aromaticity of the external effective ring of edge sites isolated for $4 k+2$ legs (aromaticity) and for odd number of legs (no aromaticity), but will not be isolated for $4 k$ legs (antiaro-

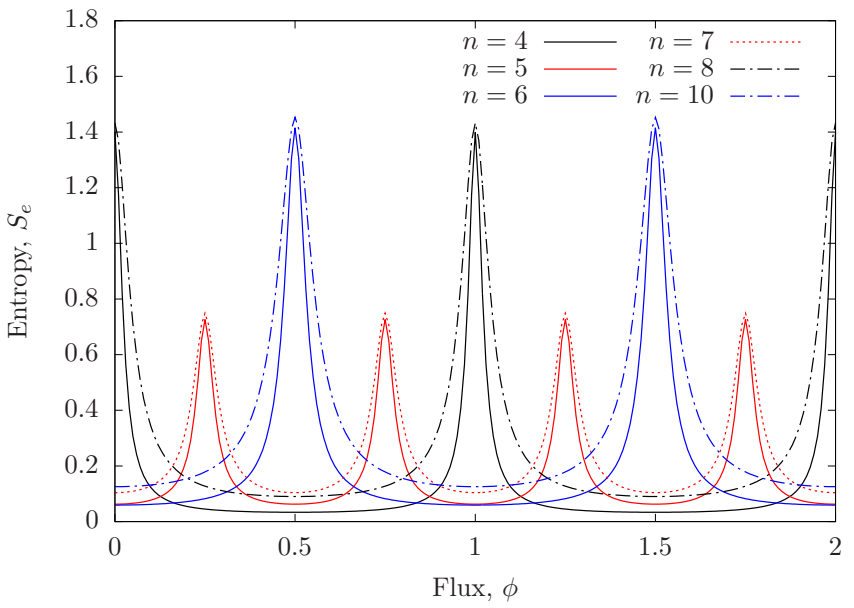

FIG. 9. Analogue of de Haas-van Alphen effect (dHvA) in junction rings. Entropy of the edge ring, $S^{B}$, as a function of the flux $\Phi$ traversing the central ring. We have used $\ell=20$ sites per leg, and $n=4,5,6,7,8$ and 10 legs. The maxima of the peaks correspond to $\log (2)$ and $2 \log (2)$.

maticity). This aromaticity effect is ubiquitous in ringed systems. 21 24]

\section{A. Magnetic Flux through Junction Rings}

The described behavior of the ring junction can change dramatically in the presence of an external magnetic field. Let $\Phi$ be the magnetic flux traversing the central ring, in units of the elementary quantum flux $\Phi_{0}$. The magnetic flux causes the hopping amplitudes to acquire a complex phase, such that the product of all phases computed clockwise will be $\exp (2 \pi i \varphi)$. Thus, we will simulate the effect of the magnetic field by including a phase $\exp (2 \pi i \varphi / n)$ in each of the couplings along the ring. Physical properties of the GS will be therefore periodic in the external flux $\Phi$, in analogy with the de Haas-van Alphen effect [25]. We will consider the effect of the magnetic flux on the edge sites, therefore an analogy with the Aharonov-Bohm effect can also be established [26.

Fig. 9 shows the entropy of the edge block, $S^{B}$, as a function of the magnetic flux crossing the central ring, for $\ell=20$ and different numbers of legs, $n$. The periodicity in $\Phi$ is perfect. Moreover, the entropy presents strong peaks at different values of the magnetic flux, which depend only on the value of $n(\bmod 4)$. For $n=4 k$, the antiaromatic junctions, we see that the entropy peaks appear at integer values of the flux $\Phi$. For $n=4 k+2$, corresponding to the aromatic junctions, the entropy peaks appear at half-integer values of the flux. In these two cases, the maximal entropy is close to $2 \log (2)$. For odd $n$, the peaks appear at $\Phi=k+1 / 4$ and $k+3 / 4$, for any integer $k$, but the maximum value is only $\log (2)$.

The physical interpretation is as follows. The GS of the $\mathrm{EH}$ presents zero modes for special values of the external 
flux, which depend on the value of $n(\bmod 4)$. For odd values of $n$, one zero mode appears at $\Phi=1 / 4$ and the other at $\Phi=3 / 4$. Thus, the degeneracy of the GS of the EH is double and $S^{B}$ peaks at $\log (2)$. For even $n$, the zero modes coincide, but they appear at different values of the magnetic flux, thus increasing the minimal entanglement entropy to $2 \log (2)$, according to Eq. (18).

In other words, for large $t_{0}$ the ring-junction system develops an edge Hamiltonian which is degenerate at specific values of the magnetic flux. Although the reduced density matrix can always be considered to be close to zero temperature, the degenerate entanglement spectra force a minimal entanglement entropy of topological origin $S_{\min }=d \log (2)$, where $d$ is the number of zero modes encountered. Thus, we see that the entropy of the edge block of ring-junctions develops a high sensitivity to the magnetic flux $\Phi$ that traverses their rings.

\section{CONCLUSIONS AND FURTHER WORK}

In this article we have explored simple quantum systems for which an increase in a few selected couplings can lead to the detachment of some block, as signaled by a vanishing entanglement entropy between the block and the rest of the system. The sites corresponding to the block need not be neighbors, and they will develop large correlations among them. We have described a few systems based on free-fermions on different quasi-1D geometries which present this entanglement detachment.

Our first example was provided by an open free fermionic chain where the second and penultimate hoppings are increased, thus detaching the block formed by the first and last sites. In the second example we detached a superlattice out of a 1D closed fermionic chain by enhancing selected hoppings. In the third and most elaborate example, we displayed the fermionic sites along a ring-junction, a star graph with a central ring, enhancing the penultimate hopping of each leg.

We have characterized the reduced density matrices of the nearly detached blocks via the entanglement en- tropy, entanglement spectrum (ES) and Hamiltonian $(\mathrm{EH})$. This way we could conclude that, both in the superlattice and the ring-junction, the $\mathrm{EH}$ present a simple scaling form for large, yet finite, enhanced hoppings. The case of the ring-junction presents an intriguing periodicity in the number of legs $n$ : anti-aromatic junctions with $n=4 k$ do not display this entanglement detachment. Instead, they have a minimal entanglement entropy equal to $2 \log (2)$. We have shown that this minimal entanglement entropy can be understood as an effect of the presence of two zero modes in the ES, which signal a non-trivial topological phase.

The topological effect becomes more interesting when we introduce an external magnetic flux through the central ring. We can observe that the entropy presents peaks of amplitude $S=d \log (2)$ with $d=1$ or 2 . The position and height of the peaks depend on the number of legs mod 4, and can be understood in terms of the zero modes of the ES and the associated topological entropy.

The systems studied can be engineered in the laboratory. These types of hopping patterns can appear naturally in quantum wires [27, 28] or organic molecules 29], or can be engineered using optical lattices employing the so-called cold-atom toolbox [30, 31. This technology is also useful, e.g., to simulate the effects of curved spacetime on quantum matter [32, 33].

Our work, thus, presents a proof-of-principle study of this entanglement detachment, but many questions are still open: the theoretical explanation of the scaling form of the entanglement Hamiltonian, the possible extension to higher dimensions, the applicability for quantum information processing and the study of dynamical effects.

\section{ACKNOWLEDGMENTS}

We would like to acknowledge very useful discussions with S.N. Santalla and G. Sierra. J.R.-L. acknowledges funding from the Spanish Government through Grant No. FIS2015-69167-C2-1-P.
[1] M.A. Nielsen, I.L. Chuang, Quantum computation and quantum information, Cambridge University Press (2000).

[2] L. Amico, R. Fazio, A. Osterloh, V. Vedral, Rev. Mod. Phys. 80, 517 (2008).

[3] J. Eisert, M. Cramer, M.B. Plenio, Rev. Mod. Phys. 82, 277 (2010).

[4] M.B. Hastings, J. Stat. Mech. P08024 (2007).

[5] G. Vitagliano, A. Riera, J.I. Latorre, New J. Phys. 12, 113049 (2010).

[6] G. Ramírez, J. Rodríguez-Laguna, G. Sierra, J. Stat. Mech. P10004 (2014).

[7] G. Ramírez, J. Rodríguez-Laguna, G. Sierra, J. Stat. Mech. P06002 (2015).
[8] H. Santos, J.E. Alvarellos, J. Rodríguez-Laguna, arXiv:1809.06246 (2018).

[9] M. Koashi, A. Winter, Phys. Rev. A 69, 022309 (2004).

[10] H. Li, F.D.M. Haldane, Phys. Rev. Lett. 101, 010504 (2008).

[11] I. Peschel, J. Phys. A: Math. Gen. 36, L205 (2003).

[12] V. Eisler, I. Peschel, J. Phys. A: Math. Theor. 50, 284003 (2017).

[13] E. Tonni, J. Rodríguez-Laguna, G. Sierra, J. Stat. Mech. 043105 (2018).

[14] Su W, Schrieffer J and Heeger A, Phys. Rev. Lett. 42, 1698 (1979).

[15] Heeger A J, Kivelson S, Schrieffer J R and Su W P, Rev. Mod. Phys. 60, 781 (1988). 
[16] J Sirker, M Maiti, N P Konstantinidis and N Sedlmayr, Journal of Statistical Mechanics: Theory and Experiment, 2014, P10032,(2014)

[17] J.K. Asbóth, L. Oroszlány, A. Pályi, A short course on topological insulators, Springer (2016).

[18] G. Vidal, J.I. Latorre, E. Rico, A. Kitaev, Phys. Rev. Lett. 90, 227902 (2003).

[19] B.T. Ye, L.Z. Mu, H. Fan, Phys. Rev. B 94, 165167 (2016).

[20] F. Pollmann, A.M. Turner, E. Berg, M. Oshikawa, Phys. Rev. B 81, 064439 (2010).

[21] S. Zilberg, Y. Haas, International Journal of Quantum Chemistry 71, 133-145 (1999).

[22] S. Wiberg, Chem. Rev. 101, 1317-1331 (2001).

[23] S. C. A. H. Pierrefixe, F. Matthias Bickelhaupt, J. Phys. Chem. A 112, 112, 12816-12822 (2008).

[24] R. Breslow, The Chemical Record 14, 1174-1182 (2014).
[25] W. J. de Haas, P. M. van Alphen, Proc. Acad. Sci. Amst. 33, 1106-1118, (1930).

[26] P.A. Orellana, M.L. Ladrón de Guevara, M. Pacheco, A. Latgé, Phys. Rev. B 68, 195321 (2003).

[27] J. R. Ahn, H. W. Yeom, H. S. Yoon, and I.- W. Lyo, Phys. Rev. Letts. 91, 196403 (2003).

[28] J. R. Ahn, P. G. Kang, K. D. Ryang, and H. W. Yeom, Phys. Rev. Letts. 95, 95, 196402, (2005).

[29] R. Grüner, Rev. Mod. Phys. 60, 1129-1181 (1988)

[30] M. Lewenstein, A. Sanpera, V. Ahufinger, Ultracold atoms in optical lattices, Oxford University Press (2012).

[31] D. Jaksch, P. Zoller, Ann. Phys. 315, 52 (2005).

[32] O. Boada, A. Celi, J.I. Latorre, M. Lewenstein, New J. Phys. 13, 035002 (2010).

[33] J. Rodríguez-Laguna, L. Tarruell, M. Lewenstein, A. Celi, Phys. Rev. A 95, 013627 (2017).

[34] S. Robles, J. Rodríguez-Laguna, J. Stat. Mech. 033105 (2017). 